\title{
Fabrication of translucent tetragonal zirconia by gelcasting of thin zirconia nano-slurry
}

\author{
Michiyuki YOSHIDA, ${ }^{\dagger}$ Shinya TAKENO and Osamu SAKURADA \\ Faculty of Engineering, Gifu University, 1-1 Yanagido, Gifu 501-1193, Japan
}

\begin{abstract}
Microstructural evolution during the sintering of nanoceramics was investigated in the compacts prepared by gelcasting of tetragonal zirconia nano-slurry with the solid loading of $7 \mathrm{vol} \%$. The initial compact prepared by the gelcasting in this study had the densely packed structure with a narrow pore-size distribution and high connectivity in the particle network. Special attentions were paid to the evolutions of pore structures in the initial and intermediate stage sintering. It was found that the coarsening of the pores, which is thought to be the important obstacle of the densification in nanoceramics, was not remarkable in the sintering of the densely packed compact. The suppression of the pore coarsening resulted in the lowering the sintering temperature. Almost fully dense sample with $99.5 \%$ of theoretical density was successfully obtained at the sintering temperature of $1373 \mathrm{~K}$ for $2 \mathrm{~h}$. The sample sintered at $1373 \mathrm{~K}$ maintained the nanometric structure with the grain size of $79 \mathrm{~nm}$ and exhibited the optical translucency.
\end{abstract}

(C2016 The Ceramic Society of Japan. All rights reserved.

Key-words : Zirconia, Gelcasting, Sintering, Nanoceramics, Microstructure, Translucency

[Received October 16, 2015; Accepted February 12, 2016]

\section{Introduction}

Since the discovery of transformation toughening, partially stabilized $\mathrm{ZrO}_{2}$ has become one of the major high-performance ceramics materials, ${ }^{1)}$ but it has received comparatively little attention with respect to its optical transmission. For noncubic ceramics, the optical anisotropy causes diffuse scattering due to birefringence at grain boundaries. Thus, the optical transparency of pore-free noncubic ceramics depends on the degree of birefringence and grain size. To improve the optical transparency in tetragonal zirconia, many efforts have been dedicated to ensure full densification with nanometric microstructure by pressure assisted sintering such as SPS ${ }^{2), 3)}$ and HIP. ${ }^{4)}$ One of the confirmed obstacles to achieve the full densification from nanosized powder is the pore coarsening. ${ }^{5)}$ As nanosized particles are much easier to move than coarse ones, the pore coalescence in nanoceramics would be greatly enhanced compared to that with micronsized grains. ${ }^{6-8)}$ The densification process of nanoceramics is greatly impeded by pore coarsening resulting from the coalescence of those interconnected pores, which involved no grain growth but particle rearrangement for accommodating space change. Exner and Muller ${ }^{9}$ have pointed out that more irregular bulk movement of particles took place in solid-state sintering of the compacts with non-uniform particle distribution, and the mobility of individual particles with the larger number of contacts was restricted. This indicates the pore coarsening is supposed to be suppressed in the compact with densely packed structure.

To eliminate agglomeration of powders and improve particles packing, colloidal processing has been developed to form

Corresponding author: M. Yoshida; E-mail: myoshida@gifu-u. ac.jp

* This article was selected from the presentations at "STAC-9" (The 9th International Conference on the Science and Technology for Advanced Ceramics) held in Tsukuba, Japan (2015), through the regular reviewing procedure. complex-shaped ceramic parts with homogeneous microstructure. ${ }^{10)}$ To take advantage of these colloidal processing techniques for shaping ceramics, it is further required to transform the fluid suspension into a rigid structure while the homogeneity of the suspension is preserved. Gelcasting was developed for this purpose where a gelling agent is added to the suspension of a ceramic powder. In the early developed gelcasting systems, acrylate monomers were mainly used as gelling agents in organic solvents. ${ }^{11)}$ Owing to the toxicity of these systems, other gelcasting systems with environmental friendly agents such as proteins ${ }^{12)}$ and polysaccharides ${ }^{13)}$ have been evaluated. For evaluation of gelcasting systems containing agar products, alumina has been the most common ceramic materials. ${ }^{14)-16)}$ Even when other gelcasting systems are considered, the use of zirconia was found to be limited ${ }^{17)}$ and this might be related to the difficulties reported in the processing of zirconia suspensions. ${ }^{18)}$

So far, preparing suspensions with high solid loading and low viscosity has always been the priority focus when the gelcasting system is of concern in sintering of ceramics. ${ }^{14), 16)}$ Because of the difficulty to obtain the near-net-shape products, less attention has been paid to the study of the gelcasting system through the suspension with low solid loadings. One of the important factors responsible for homogeneous gel structure is the distribution of the solidification agent in the suspensions. ${ }^{11)}$ Unhomogeneous gel structure results in non-uniform shrinkage during the drying, which causes defects and micro-crack. It is difficult to obtain stable slurries with a high solid loading and a low viscosity under $100 \mathrm{~nm}$ particle diameter range. ${ }^{19)}$ Colloidally stable nanoparticle suspensions are known to display a markedly lower volume loading at the same viscosity compared with suspensions with larger particle sizes. ${ }^{20)}$ This indicates the difficulty to achieve the homogeneous distribution of the geling agent to nano suspensions with higher solid loading.

In the present paper, the sintering behavior of nanocrystalline zirconia is investigated. In order to improve the optical transparency in tetragonal $\mathrm{ZrO}_{2}$, gel-casting of a dilute commercial 
nanosuspension was applied to prepare the homogeneous green body. The densely packed structure with a narrow pore-size distribution was successfully obtained by optimizing the gelcasting process. The densification behavior and the microstructural evolution during the sintering of the gelcasted compacts were studied.

\section{Experimental procedure}

In this study, the green body consisting of $3 \mathrm{~mol} \%$ yttria stabilized zirconia (3YTZ) nanoparticle was prepared by gel-casting. 3YTZ nano-slurry with the solid loading of $7.0 \mathrm{vol} \%$ (MEL Chemicals Ltd., U.K: $<0.01 \mathrm{SiO}_{2},<0.005 \mathrm{Fe}_{2} \mathrm{O}_{3}$ in wt \%) was used as the starting material for gel-casting. The solid loading of the starting slurry was measured by gravimetric analysis. The average crystallite size of the as-received slurry was $12 \mathrm{~nm}$, which correlated well with the particle size observed by TEM (Fig. 1). The as-received slurry with weak acidic pH of 2.7 was modified by adding tetra methyl ammonium hydroxide (SigmaAldrich co., llc., U.S.) to $\mathrm{pH} 9.5$ for the complete dissociation of citrate. Subsequently, $4.5 \mathrm{wt} \%$ of triammonium citrate $(99 \%$ purity, Kanto Chemical co., inc., Japan) was added as the dispersant. Agarose (LM, Kanto Chemical co., inc., Japan) was selected as the gelling agent. Agarose hydrogels can be formed from very dilute solutions (containing a fraction of one percent of agarose) and these gels have well defined shapes. Considering the process of burning out organic additives, minimizing the additive amount of agarose is preferable. After the optimization, the minimum amount of agarose necessary to form uniform gel was determined as $0.6 \mathrm{wt} \%$ based on water. The zirconia suspension was heated before adding agarose and kept at a temperature of $353 \mathrm{~K}$ in a closed vessel. To avoid an incomplete dissolution of the agarose, the zirconia suspension was stirred for an hour after adding the agarose. The prepared nano-slurriy was casted to a plastic mold with a diameter of $39 \mathrm{~mm}$, and dried in ambient temperature.

The green bodies were sintered at the temperature between 1173 and $1473 \mathrm{~K}$ for $2 \mathrm{~h}$ in an air atmosphere. Based on TG/DTA analysis, organic additives were almost decomposed at the temperature of $673 \mathrm{~K}$. To avoid the propagation of the micro-cracks due to the decomposition of organic additives, the green bodies were heated gently at the ramp rate of $0.2 \mathrm{~K} / \mathrm{min}$ to $923 \mathrm{~K}$, and then the ramp rate was set to $1 \mathrm{~K} / \mathrm{min}$. Relative density was calculated using archimedes method by taking $6.10 \mathrm{~g} / \mathrm{cm}^{3}$ as the theoretical density of 3 Y-TZP. The specific surface area and total pore volume of samples in the initial stage of sintering (with open porosity) were measured by the multipoint nitrogen adsorption BET method (Model NOVA 2200e, Quantachrome Co., U.S.). The pore size distribution of these samples was determined according to the BJH theory. Based on the results of the multipoint nitrogen adsorption, the grain size in the initial stage

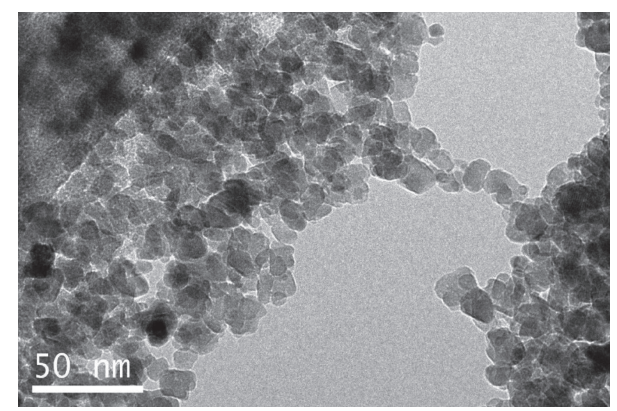

Fig. 1. Transmission electron microscopy image of the $3 \mathrm{~mol} \%$ yttria stabilized zirconia nanoparticles. of sintering was calculated by using the following equation,

$$
d=\frac{6}{\rho \cdot S}
$$

Where $d$ is the diameter, $\rho$ is the density and $S$ is the specific surface area. Pore morphologies and grain size in the samples were studied by cross-sectional image. Samples sintered under $1173 \mathrm{~K}$, mainly consist of open pores, were incorporated into epoxy resin and cut by low speed diamond saw. The cross-section of the embedded samples was polished by Ar ion beam. The samples sintered over $1273 \mathrm{~K}$ with higher densities were mechanically polished and thermally etched to have surface grooves at the grain boundaries. The microstructure of the osmium coated samples was observed with scanning electron microscope (Model S4800, Hitachi Co., Japan). The equivalent circle diameter measured by the image analysis of SEM photographs was defined as the grain size.

\section{Results}

Photograph of the gelcasted compact after drying is shown in Fig. 2. The low solid loading of the slurry resulted in a large drying shrinkage. After complete drying, the linear shrinkage of the sample was $43.5 \%$. No micro cracking and uniform shrinkage in a radial direction were observed. Figure 3 shows the relative density as a function of the sintering temperature. To evaluate the microstructure during the initial and intermediate stage of sintering, samples were immediately cooled after reaching the temperature of $673,923,1073$ and $1173 \mathrm{~K}$. The relative density sintered at $1173,1273,1373$ and $1473 \mathrm{~K}$ for $2 \mathrm{~h}$ are plotted in Fig. 3 together with the immediately cooled samples. The rela-

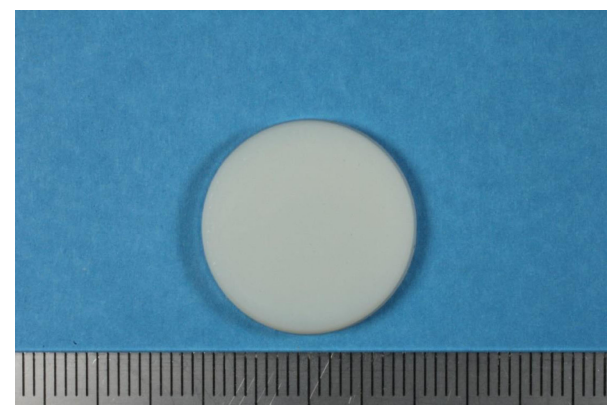

Fig. 2. Photograph of the gelcasted compact.

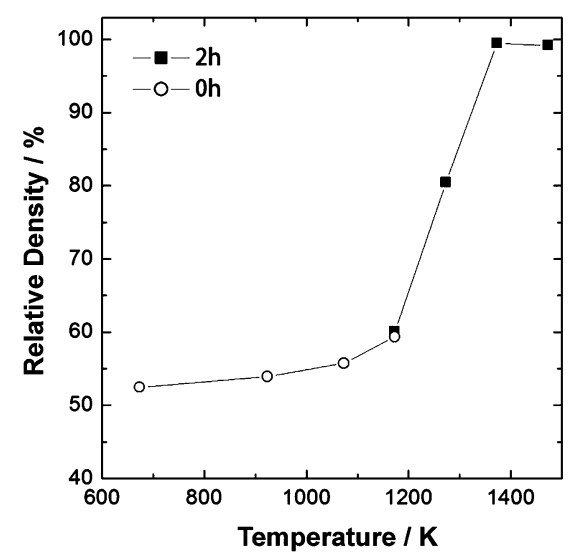

Fig. 3. Relative density as a function of sintering temperature. Open circles show data for immediately cooled sample. Solid squares show the density of sample sintered for $2 \mathrm{~h}$. 


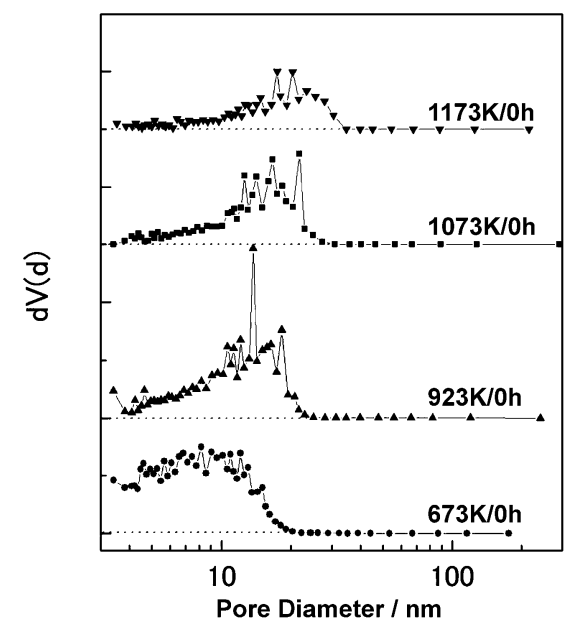

Fig. 4. Evolution of pore size distribution for gelcasted 3YTZP (horizontal dashed lines indicate zero point for every distribution curve).

tive density was gradually increased until $1173 \mathrm{~K}$, and increased rapidly from 1173 to $1373 \mathrm{~K}$. Almost fully dense sample with $99.5 \%$ of theoretical density was obtained at the sintering temperature of $1373 \mathrm{~K}$ for $2 \mathrm{~h}$. The densities for the sample sintered at $1173 \mathrm{~K}$ for 0 and $2 \mathrm{~h}$ were 59 and $60 \%$, respectively. This indicates that the density was not so much affected by the holding time below the temperature of $1173 \mathrm{~K}$. The initial and intermediate stages of sintering are investigated by nitrogen adsorption measurements. The evolution of the pore size distributions for gelcasted compacts is summarized in Fig. 4, where the distribution of the logarithm of the pore size evaluated by $\mathrm{BJH}$ method from desorption isotherms is plotted for the temperature of $673,923,1073$ and $1173 \mathrm{~K}$. In the sample sintered at $673 \mathrm{~K}$, distribution profile of pore size showed the skewed distribution which trailed to smaller pore size. As increasing the temperature, pores smaller than $10 \mathrm{~nm}$ shrank, while the shrinkage of larger pore was not noticeable. The average pore size slightly increased from $9.9 \mathrm{~nm}$ at $673 \mathrm{~K}$ to $13.7 \mathrm{~nm}$ at $923 \mathrm{~K}$, $16.3 \mathrm{~nm}$ at $1073 \mathrm{~K}$ and $20.3 \mathrm{~nm}$ at $1173 \mathrm{~K}$. The pore growth factor is defined as $D_{t} / D_{0}$, where $D_{0}$ and $D_{t}$ are pore diameters in the initial stage and at the time (or stage) of concern, respectively. Pore growth factor of the initial and intermediate stage sintering in this study was 2.05 , which was smaller than the reported value of 9 in the sintering of nanocrystalline zirconia. ${ }^{7)}$ Figure 5 shows FE-SEM micrographs for the cross section in the compact prepared by gel-casting of zirconia nano-slurry. In the cross section of the sample sintered at $923 \mathrm{~K}$, the densely packed structure was observed. The observed pores had the size under $30 \mathrm{~nm}$ which was in good agreement with the result from BJH method. They were in the size range of the raw material and distributed homogeneously in the compacts. As increasing the sintering temperature, pores grew slightly, but still maintain the size under $50 \mathrm{~nm}$. The curtain effect shown in the cross section of the sample sintered at $1073 \mathrm{~K}$ is an artifact often observed on ion beam polished surface as thickness striations. As seen in the results of the nitrogen adsorption measurement and the microstructural observation, the coarsening of the pores during the initial and intermediate stage sintering was not remarkable. Average grain size and average pore size are plotted as a function of sintering temperature in Fig. 6. The pore size and the grain size increased with increasing the temperature, but the average pore size remains under the grain size in the temperature range from 673 to $1173 \mathrm{~K}$. Two distinct regions showing different grain
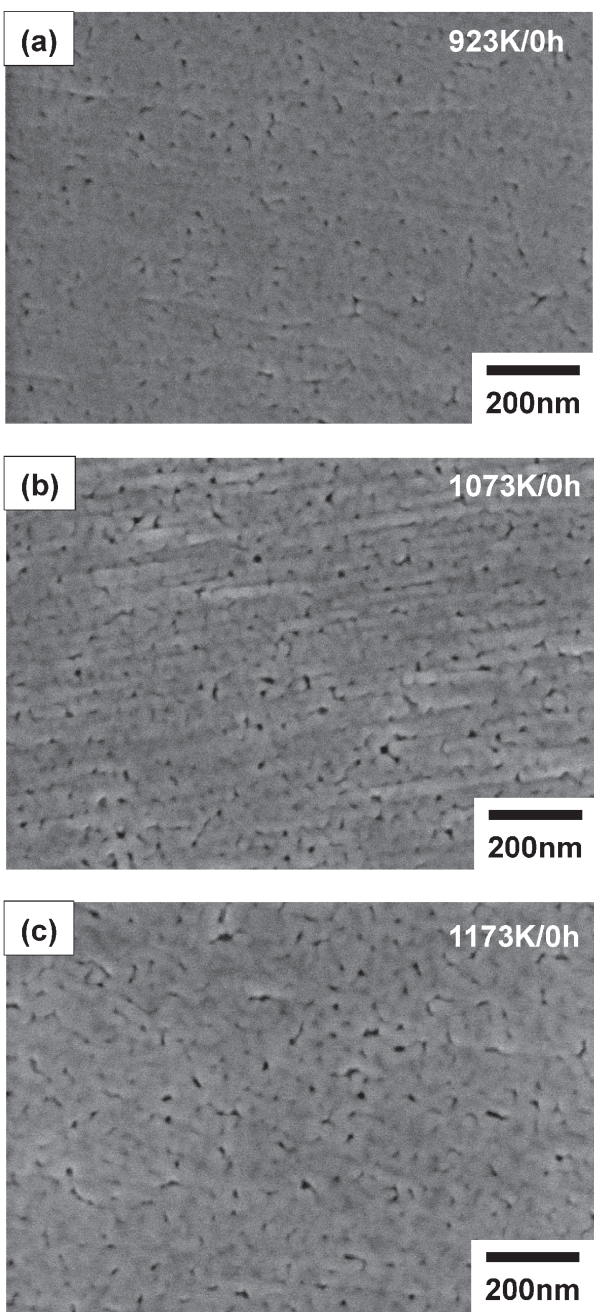

Fig. 5. Cross sectional images for gelcasted 3YTZP sintered at different temperatures: (a) $923 \mathrm{~K}$, (b) $1073 \mathrm{~K}$, (c) $1173 \mathrm{~K}$.

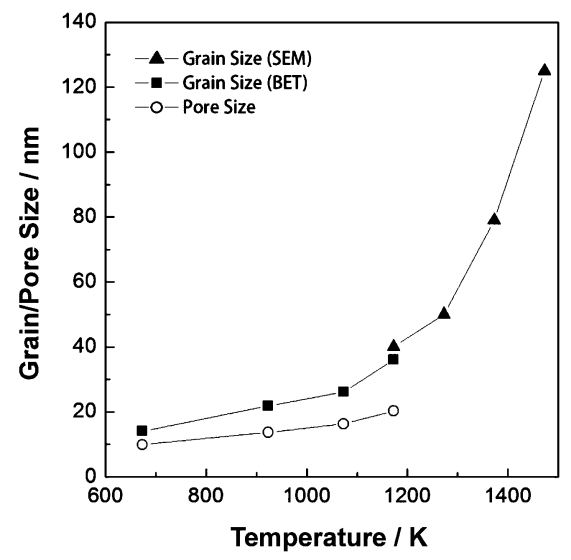

Fig. 6. Grain size and pore size as a function of sintering temperature. Solid squares show grain size calculated from the results of the multipoint nitrogen adsorption. Solid triangles indicate the grain size determined by image analysis of SEM photographs.

growth behavior are observed in Fig. 6. The grain size gradually increased up to around $90 \%$ of theoretical. The considerable grain growth was observed at densities higher than $90 \%$. The transition from gradual grain growth to rapid grain growth at around $90 \%$ density is believed to change from open to closed porosity. SEM 

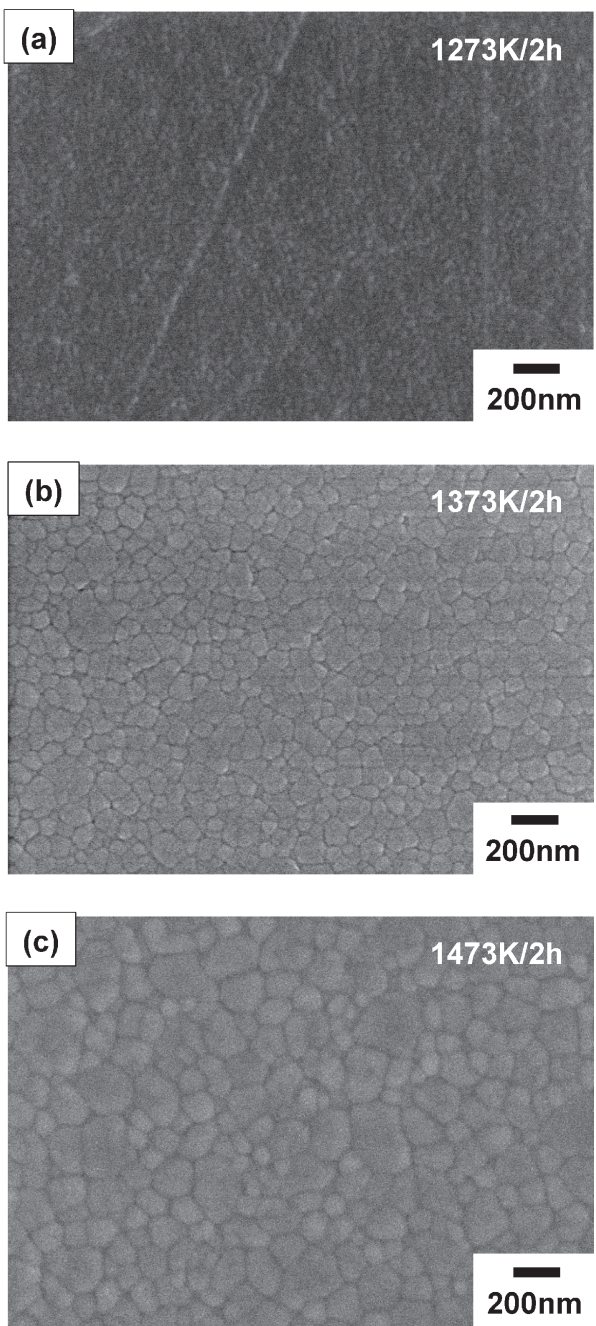

Fig. 7. SEM micrographs of thermal etched surfaces of gelcasted 3 YTZP sintered at different temperatures for $2 \mathrm{~h}$ : (a) $1273 \mathrm{~K}$, (b) $1373 \mathrm{~K}$, (c) $1473 \mathrm{~K}$.

micrographs of the gelcasted samples sintered at 1273, 1373 and $1473 \mathrm{~K}$ are shown in Fig. 7. As increasing the sintering temperature, coarsening in the microstructure took place, but the structural uniformity was preserved. The relative density of the sample sintered at $1273 \mathrm{~K}$ with the grain size of $50 \mathrm{~nm}$ was $80 \%$. At higher magnification of the sample sintered at $1273 \mathrm{~K}$, the presence of pores smaller than $50 \mathrm{~nm}$ was observed. These Pores distributed homogeneously in the microstructure. The microstructure of the sample sintered at $1373 \mathrm{~K}$ maintained the nanometric structure with grain size of $79 \mathrm{~nm}$. There is no exaggerated grain growth, and the fully dense sample with a density of $99.5 \%$ was obtained. In the sample sintered at $1473 \mathrm{~K}$, coarsening was observed and grain size increased to have the value of $125 \mathrm{~nm}$. The success in retaining nanometric structures while achieving full densification meets the conditions for increasing optical transparency of noncubic ceramics. To confirm the optical transparency, the sintered sample was grinded to have the thickness of $0.6 \mathrm{~mm}$, and then both surfaces of the grinded samples were mirror-polished using diamond slurry. Figure 8 shows the appearance of the samples sintered at 1273, 1373 and $1473 \mathrm{~K}$. The accordingly sintered TZP with nanoceramic structure exhibited the optical translucency, not to say transparency. The samples sintered at $1273 \mathrm{~K}$ are not fully dense. The presence

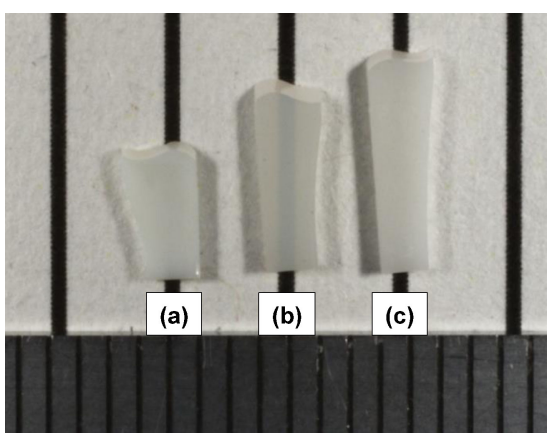

Fig. 8. The appearance of the sintered samples of $0.6 \mathrm{~mm}$ in thickness: (a) $1273 \mathrm{~K}$, (b) $1373 \mathrm{~K}$, (c) $1473 \mathrm{~K}$

of pores cause intensive light scattering that turn the sintered body to opaque. The almost pore free sample sintered at $1373 \mathrm{~K}$ with grain size of $79 \mathrm{~nm}$ showed optical translucency. Further increase of sintering temperature resulted in grain growth, and the sample sintered at $1473 \mathrm{~K}$ showed opaque due to diffuse scattering at grain boundaries.

\section{Discussion}

Some advantages have been reported in the synthesis of translucent tetragonal $\mathrm{ZrO}_{2}$ by pressure assisted sintering. ${ }^{2)-4)}$ According to Trunec et al., ${ }^{21)}$ the pore coarsening occurring in the initial and intermediate sintering stage could be suppressed by applying a uniaxial pressure. During the initial stage sintering, where grain sliding dominates the sintering process, particle rearrangement enhanced by the use of an external force could break large pores. The enhancement of the densification rate by retaining finer pore size during sintering results in the reduction of the sintering temperature. The lowering of the sintering temperature suppresses the grain growth, and thus the sample prepared by the pressure assisted sintering showed the improved optical translucency. In this study, the pore coarsening during the initial stage sintering was inhibited without applying a uniaxial pressure. Kingery ${ }^{22)}$ proposed the criterion of the pore stability in ceramics, where small pores with concave faces tend to shrink, while large pores with convex faces tend to grow. The critical pore size is defined as the size of a stable pore with flat faces and can be determined if the particle size and dihedral angle are known. Typical values of a dihedral angle for oxide ceramics are in the range from 100 to $150^{\circ}{ }^{6}{ }^{6,22)}$ Assuming an average value for the dihedral angle, the critical pore sizes of the $\mathrm{ZrO}_{2}$ is supposed to be the order of the grain sizes. Figure 6 shows the pore size and the grain size as a function of sintering temperature. Although both pore size and grain size increased with increasing temperature, the average pore size remains in the range of the grain size. Comparing the pore size distribution of samples sintered at $673 \mathrm{~K}$ (with a critical pore size estimated to be $10 \mathrm{~nm}$ ) and $1173 \mathrm{~K}$, it can be seen that almost all pores smaller than the critical pore size disappeared in the sample sintered at $1173 \mathrm{~K}$ (Fig. 4). Like the coarsening of solid particles, the driving force behind the pore coarsening can be understood in terms of minimizing the surface energy. During sintering, the reduction of the total surface energy can be achieved by the reduction of total pore volume or geometric changes of the solid/porosity interface. The latter is usually related to the origin of pore coarsening. The kinetics of this process is, however, quite complicated. Among all the possible factors, the pore size, the pore size distribution, the gas inclusion inside the pores, and the temperature are thought to have strong influences on the kinetics of pore coarsening. The 
application of gelcasting, in order to obtain green bodies with a homogeneous structure resulted in inhibiting the pore coarsening. The initial compact prepared by the gelcasting in this study had the densely packed structure with a narrow pore-size distribution and high connectivity in the particle network. This homogeneous structure is thought to prevent the pore coarsening during initial and intermediate stage of sintering.

In general, it is a key step to attain high solid loading suspensions for achieving high density of gelcasted compacts without drying and sintering-induced defects, such as cracking and warping. ${ }^{11)}$ In this study, the densely packed structure was successfully obtained through the gelcasting of the thin slurry with the solid loading of $7 \mathrm{vol} \%$. The results shown in this study indicate a high solid loading is not always required as often claimed for direct consolidation methods in order to reach high sintered density. The key factors to achieve high density of green body in gelcasting from thin nano particle slurry are thought to be: (1) gentle drying, (2) fluidity of the slurry and (3) appropriate strength of the gel network.

In the initial drying stage of gelcasted compact, the moisture, in the form of liquid water, is transported primarily via capillary forces to the surface of the part, where it evaporates to the atmosphere. ${ }^{23)}$ The gel matrix yields to the large compressive stress that are set up by the capillary forces. The particles, which are captured in the hydrogel, move toward each other under the compressive stress, which, in turn, causes a bulk shrinkage of the compact. Although the moisture-distribution in the initial green body is homogeneous, the moisture gradient, which is attributed to the difference in the rate of the moisture drains between the surface and the center of the wet green body, arises during the drying. The higher moisture gradient makes the green body shrink unevenly, and then inner stress occurs. In this study, gentle condition of drying at room temperature decreased the moisture gradient in the wet green body, and resulted in the homogeneous shrinkage during drying.

As advancing the drying process, the mean inter-particle distance in the hydrogel becomes shorter. In this case, interaction between particles is thought to be similar as the concentrated slurry. If the fluidity of the particles in the hydrogel is not enough to have the rearrangement under the large compressive stress originated by capillary forces, the inner stress will arise and the bulk shrinkage of the wet green body becomes uneven. In our first trial of this process, the wet green body prepared from the slurry without the surfactant broke to pieces during drying. I. Santacruz et al. ${ }^{24)}$ successfully developed dense $3 \mathrm{~mol} \%$ yttriastabilized tetragonal zirconia (YTZ) through slip-casting of the concentrated nano-slurry. They reported that the triammonium citrate (TAC) acted as an anionic dispersant to zirconia nanoparticles dispersed in deionized water. Our results indicated that TAC was also effective as a dispersant of zirconia nanoparticles in agarose hydrogel.

Agarose adopts a single conformation in the water solution over the temperature of $353 \mathrm{~K}$. As cooling the water solution, gelation occurs from aggregation of double helices at a temperature around 293-303 K. This three-dimensional network based on association of double helices is stabilized by cooperative hydrogen bonds. ${ }^{25}$ ) The average pore diameter in agarose hydrogel depends on the amount of the geling agent, and the reported pore diameter ranges 200 to $500 \mathrm{~nm} .{ }^{26), 27)}$ The strength of the agarose hydrogel depends on the macromolecular network in the hydrogel. ${ }^{28)}$ When the strength of the macromolecular network is sufficient to choke back the capillary forces, the inner stress is caused by the uneven shrinkage of the wet green body. In the gelcasting of thin zirconia nano slurry with the solid loading of $7 \mathrm{vol} \%$, the addition of the higher amount of agarose (over $1 \mathrm{wt} \%$ ) resulted in the propagation of micro crack during the drying of the wet green body. Although a prolonged drying time is the drawback of this process, using the slurry with lower solid loading is effective to achieve the densely packed structure with nano particles.

\section{Conclusions}

Microstructural evolution during the sintering of nanoceramics was investigated in 3Y-TZP compacts prepared by gelcasting of zirconia nano-slurry with the solid loading of $7 \mathrm{vol} \%$. The gently dried compact exhibited the densely packed structure with a narrow pore-size distribution and high connectivity in the particle network. The coarsening of the pores, which is often thought to be the important obstacle of the densification in nanoceramics, was not remarkable in the sintering of the densely packed compact. The suppression of the pore coarsening resulted in the lowering the sintering temperature. Almost fully dense sample with the grain size of $79 \mathrm{~nm}$ was successfully obtained at the sintering temperature of $1373 \mathrm{~K}$. The success in retaining nanometric structures while achieving full densification meets the conditions for increasing optical transmission of noncubic ceramics, and the sample sintered at $1373 \mathrm{~K}$ exhibited the optical translucency.

\section{References}

1) J. Chevalier, L. Gremillard, A. V. Virkar and D. R. Clarke, J. Am. Ceram. Soc., 92, 1901-1920 (2009).

2) S. R. Casolco, J. Xu and J. E. Garay, Scr. Mater., 58, 516-519 (2008).

3) U. Anselmi-Tamburini, J. N. Woolman and Z. A. Munir, $A d v$. Funct. Mater., 17, 3267-3273 (2007).

4) J. Klimke, M. Trunec and A. Krell, J. Am. Ceram. Soc., 94, 1850-1858 (2011).

5) J. A. Varela, O. J. Whittemore and E. Longo, Ceram. Int., 16, 177-189 (1990).

6) P. L. Chen and I. W. Chen, J. Am. Ceram. Soc., 79, 3129-3141 (1996).

7) V. V. Srdić, M. Winterer and H. Hahn, J. Am. Ceram. Soc., 83, 1853-1860 (2000).

8) Y. Xiong, J. Hu, Z. Shen, V. Pouchly and K. Maca, J. Am. Ceram. Soc., 94, 4269-4273 (2011).

9) H. E. Exner and C. Müller, J. Am. Ceram. Soc., 92, 1384-1390 (2009).

10) C. Tallon and G. V. Franks, J. Ceram. Soc. Japan, 119, 147160 (2011).

11) J. Yang, J. Yu and Y. Huang, J. Eur. Ceram. Soc., 31, 25692591 (2011).

12) O. Lyckfeldt, J. Brandt and S. Lesca, J. Eur. Ceram. Soc., 20, 2551-2559 (2000).

13) A. J. Fanelli, R. D. Silvers, W. S. Frei, J. V. Burlew and G. B. Marsh, J. Am. Ceram. Soc., 72, 1833-1836 (1989).

14) Z. P. Xie, J. L. Yang, D. Huang, Y. L. Chen and Y. Huang, $B r$. Ceram. Trans., 98, 58-61 (1999).

15) A. J. Millan, R. Moreno and M. I. Nieto, Mater. Lett., 47, 324 328 (2001).

16) I. Santacruz, M. I. Nieto and R. Moreno, Ceram. Int., 31, 439445 (2005).

17) E. Adolfssonw, J. Am. Ceram. Soc., 89, 1897-1902 (2006).

18) M. L. Rami, M. Meireles, B. Cabane and C. Guizard, J. Am. Ceram. Soc., 92, S50-S56 (2009).

19) H. B. Shan and Z. T. Zhang, Br. Ceram. Trans., 95, 35-38 (1996).

20) L. P. Meier, L. Urech and L. J. Gauckler, J. Eur. Ceram. Soc., 24, 3753-3758 (2004). 
21) M. Trunec, K. Maca and Z. J. Shen, Scr. Mater., 59, 23-26 (2008).

22) W. D. Kingery, H. K. Bowen and D. R. Uhlmann, "Introduction to Ceramics", Wiley, New York, (1976).

23) S. Ghosal, A. E. Naeini, Y. P. Harn, B. S. Draskovich and J. P. Pollinger, J. Am. Ceram. Soc., 82, 513-520 (1999).

24) I. Santacruz, K. Anapoorani and J. Binner, J. Am. Ceram. Soc., 91, 398-405 (2008).
25) P. L. S. Biagio, F. Madonia, J. Newman and M. U. Palma, Biopolymers, 25, 2255-2269 (1986).

26) M. M. Chui, R. J. Phillips and M. J. McCarthy, J. Colloid Interface Sci., 174, 336-344 (1995).

27) M. Maaloum, N. Pernodet and B. Tinland, Electrophoresis, 19, 1606-1610 (1998).

28) V. Normand, D. L. Lootens, E. Amici, K. P. Plucknett and P. Aymard, Biomacromolecules, 1, 730-738 (2000). 